\title{
HYPERFINITE AND STANDARD UNIFICATIONS FOR PHYSICAL THEORIES
}

\author{
ROBERT A. HERRMANN
}

(Received 1 March 2001)

\begin{abstract}
A set of physical theories is represented by a nonempty subset $\left\{S_{N_{j}}^{V} \mid j \in \mathbb{N}\right\}$ of the lattice of consequence operators defined on a language $\Lambda$. It is established that there exists a unifying injection $\mathscr{S}$ defined on the nonempty set of significant representations for natural systems $M \subset \Lambda$. If $W \in M$, then $\mathscr{S}_{W}$ is a hyperfinite ultralogic and $\bigcup\left\{\mathbf{S}_{\mathbf{N}_{j}}^{\mathbf{V}}(\mathbf{W}) \mid j \in\right.$ $\mathbb{N}\}=\mathscr{S}_{W}\left({ }^{*} \mathbf{W}\right) \cap \Lambda$. A "product" hyperfinite ultralogic $\Pi$ is defined on internal subsets of the product set ${ }^{*} \Lambda^{m}$ and is shown to represent the application of $\mathscr{S}$ to $\left\{W_{1}, \ldots, W_{m}\right\} \subset M$. There also exists a standard unifying injection $S_{W}$ such that $\mathscr{S}_{W}\left({ }^{*} \mathbf{W}\right) \subset{ }^{*} \mathbf{S}_{\mathbf{W}}\left({ }^{*} \mathbf{W}\right)$.
\end{abstract}

2000 Mathematics Subject Classification. 03G10, 03H10, 03B80.

1. Introduction. As discussed in [7], Tarski [8, pages 60-109] introduced consequence operators as models for various aspects of human thought. Within lattice theory, there are two such mathematical theories investigated, the general and the finitary consequence operators [2]. Let $L$ be a nonempty language, $\mathscr{P}$ the power set operator, and $\mathscr{F}$ the finite power set operator.

DEFINITION 1.1. A mapping $C: \mathscr{P}(L) \rightarrow \mathscr{P}(L)$ is a general consequence operator (or closure operator) if for each $X, Y \in \mathscr{P}(L)$

(i) $X \subset C(X)=C(C(X)) \subset L$; and if

(ii) $X \subset Y$, then $C(X) \subset C(Y)$.

A consequence operator $C$ defined on $L$ is said to be finitary if it satisfies

(iii) $C(X)=\bigcup\{C(A) \mid A \in \mathscr{F}(X)\}$.

REMARK 1.2. The above axioms (i), (ii), and (iii) are not independent. Indeed, (i), (iii) imply (ii). The phrase "defined on $L$ " means formally defined on $\mathscr{P}(L)$.

2. Axiomatic consequence operators. Prior to simplification, assume that our consequence operators are axiomatic, where the axioms include appropriate natural laws or processes. As done in [5, page 12], only consider equivalent representatives as the members of $L$. Let $\mathscr{C}(L)$ (resp., $\left.\mathscr{C}_{f}(L)\right)$ be the set of all general (resp., finitary) consequence operators defined on $L$, where $A \subset L$ is the set of logical axioms for $F \in \mathscr{C}(L)$ (resp., $\mathscr{C}_{f}(L)$ ).

The use of axiomless operators (see [2, page 3]) leads to an important simplification. For $F \in \mathscr{C}(L)$ (resp., $\mathscr{C}_{f}(L)$ ), let $A \cup N \subset L$ and suppose that $F(\varnothing) \supset A \cup N$. (Note that $N$ does not denote the natural numbers.) Then $\varnothing \subset A \cup N$ yields $F(\varnothing) \subset F(A \cup N)$, and $A \cup N \subset F(\varnothing)$ yields that $F(A \cup N) \subset F(F(\varnothing))=F(\varnothing)$. Hence, $F(\varnothing)=F(A \cup N)$. 
Further, if $B \subset A \cup N$, then since $\varnothing \subset B$, it follows that $F(\varnothing)=F(A \cup N) \subset F(B) \subset$ $F(F(A \cup N))=F(A \cup N)$ and $F(B)=F(A \cup N)$. The objects in $F(A \cup N)$ behave as if they are axioms for $F$. Can this axiomatic behavior be used to generate formally a specific consequence operator $C$, where $C(\varnothing)=\varnothing$, and the only results displayed by this model are conclusions not members of $F(A \cup N)$ ? If such a meaningful consequence operator exists, then this approach is acceptable since if natural laws or processes, as represented by $N$, are stated correctly, such as always including any physical circumstances that might restrict their application, then they behave like physical "tautologies" for our universe. For such a basic consequence operator $F$, the set $F(\varnothing)$ is composed of all of the restatements of $N$ that are considered as "logically" equivalent, and all of the pure "logical" theorems.

In general, various forms of scientific argument are modeled by consequence operators. The axioms are but inserted into an argument after which the actual rules of inference are applied that might yield some $x \in L-F(\varnothing)$. It is this $x$ that may yield something not trivial. In the physical case, this $x$ may represent some aspect of an actual physical object distinct from the natural laws or processes.

3. Rules that generate consequence operators. In this investigation, the term "deduction" is broadly defined. Informally, the pre-axioms $A \cup N$ form a subset of our language $L$, where $N$ represents natural laws or processes, and there exists a fixed finite set $\mathbf{R I}=\left\{R_{1}, \ldots, R_{p}\right\}$ of $n$-ary relations $(n \geq 1)$ on $L$. The term "fixed" means that no member of RI is altered by any set $X$ of hypotheses that are used as discussed below. (It is possible that some of these $R_{i}$ are $N$ dependent.) It can be effectively decided when an $x \in L$ is a member of $A \cup N$ or a member of any of the fixed 1-ary relations. Further, for any finite $B \subset L$ and a $(j+1)$-ary $R_{i} \in \mathbf{R I}, j>1$, and any $f \in R_{i}$, it is assumed that it can be effectively decided whether the $k$ th coordinate value $f(k) \in B$, $k=1, \ldots, j$. It is further assumed that a mental or equivalent activity called deduction from a set of hypotheses can be represented by a finite (partial) sequence of numbered (in order) steps $b_{1}, \ldots, b_{m}$ with the final step $b_{m}$ the conclusion of the deduction. All of these steps are considered as represented by objects from the language $L$. Any such representation is composed either of the zero step, indicating that there are no steps in the representation, or one or more steps with the last numbered step being some $m>0$. In this inductive step-by-step construction, a basic rule used to construct this representation is the insertion rule. If the construction is at the step number $m \geq 0$, then the insertion rule, $\mathbf{I}$, is the "insertion of a hypothesis from $X \subset L$, or insertion of a member from the set $A \cup N$, or the insertion of any member of any 1-ary relation, and denoting this insertion by the next step number." If the construction is at the step number $m>0$, then the rules of inference, RI, are used to allow for an insertion of a member from $L$ as a step number $m+1$, in the following manner. For any $(j+1)$-ary $R_{i} \in \mathbf{R I}, 1 \leq j$, and any $f \in R_{i}$, if $f(k) \in\left\{b_{1}, \ldots, b_{m}\right\}, k=1, \ldots, j$, then $f(j+1)$ can be inserted as a step number $m+1$. Note, in particular, how specific "choices" are an essential part of the process here termed as deduction.

There may exist special binary relations $\mathbf{J}$ that are members of RI. These relations are identity styled relations in that the first coordinate and second coordinates are identical. In scientific theory building, these are used to indicate that a particular 
set of natural laws or processes does not alter a particular premise that describes a natural-system characteristic. The statement represented by this premise remains part of the final conclusion. Scientifically this can be a significant fact. The deduction is constructed only from the rule of insertion or the rules of inference as described in this and the previous paragraph.

If you apply these procedures to obtain the final step as your deduction, then these procedures are modeled by a finitary consequence operator. For the language $L$, a set of pre-axioms $A \cup N$, a set RI and any $X \subset L$, define the set map $C_{N}$, by letting $C_{N}(X)$ be the set of all members of $L$ that can be obtained from $X$ by "deduction." Clearly, by insertion $X \subset C_{N}(X)$. Since $C_{N}(X) \subset L$, then consider the statement $C_{N}\left(C_{N}(X)\right)$. Since no member of the set RI is altered by introducing a different set of hypotheses such as $C_{N}(X)$, then this composition is defined. Let $x \in C_{N}\left(C_{N}(X)\right)$. By definition, $x$ is the final step in a finite list $\left\{b_{i}\right\}$ of members from $L$. The steps in this finite "deduction" from which $x \in L$ is obtained are the I steps, where added to these insertions are only members from $C_{N}(X)$, while the RI steps, as defined above, are fixed. Suppose that $b_{i} \in$ $C_{N}(X)$ is any of these additional insertions. Simply construct a new finite sequence of steps by substituting for each such $b_{i}$ the finite sequence of steps from which $b_{i}$ is the final step in deducing that $b_{i} \in C_{N}(X)$. The resulting finite collections of steps are then renumbered. The final step in this new finite deduction is $x$. Since the reasons for all of the steps are either the original I or RI, and RI contains predetermined $n$ ary relations that are not dependent upon any deduction, then the finite sequence obtained in this manner is a deduction for a member of $C_{N}(X)$. Hence, $x \in C_{N}(X)$. Consequently, $C_{N}\left(C_{N}(X)\right)=C_{N}(X)$. The finitary requirement is obvious since there are only a finite number of steps in any deduction. Note that $C_{N}(\varnothing) \supset B$, where $B$ is the set of all $x \in L$ such that $x$ is a step obtained only by the rule $\mathbf{I}$. Throughout the remainder of this paper, it is assumed that all "deductions" follow these procedures and the corresponding consequence operator is defined as in this paragraph.

4. Intrinsic natural laws or processes. For "scientific deduction" for a fixed science-community, $i$, consider as our rules of inference a collection $\mathbf{R}_{\mathbf{i}}=\mathbf{R I}$ of all of the "rules of inference used by this specific scientific-community and allowed by their scientific method" as they are applied to a specified language $\Sigma_{i}$, the language for "their science." At present, this definition for $\mathbf{R}_{\mathbf{i}}$ is rather vague. Hence, the existence of such a set $\mathbf{R}_{\mathbf{i}}$, the rules of inference for a science-community, is an assumption. From this, a specific "science" consequence operator $S_{N_{i}}$ is generated for each set of pre-axioms $A_{i} \cup N_{i}$, where $A_{i}$ are the basic logical axioms and $N_{i}$ the natural laws or processes. For proper application, the science consequence operator is applied to specific natural-systems, not those generally described. Thus $S_{N_{i}}$ has physical meaning only when $S_{N_{i}}$ is applied to an $X$ where every member of $X$ and $S_{N_{i}}(X)$ is a "tagged" statement that identifies a specific natural-system [1].

The axiomatic consequence operator $S_{N_{i}}: \mathscr{P}\left(\Sigma_{i}\right) \rightarrow \mathscr{P}\left(\Sigma_{i}\right)$, where $S_{N_{i}}(\varnothing) \supset\left(A_{i} \cup\right.$ $N_{i}$ ), can be reduced, formally, to an axiomless consequence operator on the language $\Sigma_{i}-S_{N_{i}}\left(A_{i} \cup N_{i}\right)$ as shown by Tarski [8, page 67]. In this paper, this single process is termed relativization. Let $V=\left\{A_{i}, N_{i}\right\}$. For each $X \subset \Sigma_{i}-S_{N_{i}}\left(A_{i} \cup N_{i}\right)$, let $S_{N_{i}}^{V}(X)=$ $\left(\Sigma_{i}-S_{N_{i}}\left(A_{i} \cup N_{i}\right)\right) \cap S_{N_{i}}(X)$. For this $S_{N_{i}}$, the operator $S_{N_{i}}^{V}$ is a consequence operator 
on $\Sigma_{i}-S_{N_{i}}\left(A_{i} \cup N_{i}\right)$ and has the property that $S_{N_{i}}^{V}(\varnothing)=\varnothing$. Thus, using $S_{N_{i}}\left(A_{i} \cup N_{i}\right)$ as a set of axioms, logical and physical, $S_{N_{i}}^{V}$ behaves as if it is axiomless, where the explicit natural laws or processes $N_{i}$ behave as if they are implicit. Since, in general, $S_{N_{i}}\left(A_{i} \cup N_{i}\right) \subset S_{N_{i}}(X)$, the only consequences that are not but specific deductions from the pre-axioms $A_{i} \cup N_{i}$ are members of $S_{N_{i}}(X)-S_{N_{i}}\left(A_{i} \cup N_{i}\right)$, where the explicit $X$ should not include members of $S_{N_{i}}\left(A_{i} \cup N_{i}\right)$. Physically, $S_{N_{i}}^{V}$ is the exact operator that, using implicitly such axioms as $S_{N_{i}}\left(A_{i} \cup N_{i}\right)$, characterizes the coalescing of a given fundamental collection of named and tagged objects in $X$ and that creates a different natural-system or that alters natural-system behavior. The use of axiomless consequence operators is an important simplification.

5. A hyperfinite unification for physical theories. Although all that follows can be applied to arbitrary science-communities, for notational convenience, consider but one science-community. Assume that there is one language for science $\Sigma$ and one sequentially represented countable family of natural laws or processes and logical axioms $A_{j} \cup N_{j}$ as well as one family of sequentially represented rules of inference $\mathbf{R}_{\mathbf{j}}$ that generate each specific theory. Let the sequentially represented $V=\left\{A_{j} \cup N_{j} \mid\right.$ $j \in \mathbb{N}$ \}. This yields the sequentially represented countable set of physical theories $\left\{S_{N_{i}} \mid j \in \mathbb{N}\right\}$ and the countable set $\left\{S_{N_{j}}^{V} \mid j \in \mathbb{N}\right\}$ of intrinsic sequentially represented consequence operators defined on $\Sigma-\left(\bigcup\left\{S_{N_{j}}\left(A_{j} \cup N_{j}\right) \mid j \in \mathbb{N}\right\}\right)=\Lambda$.

DEFINITION 5.1. A nonempty $X \in \mathscr{P}(\Lambda)$ is called a significant member of $\mathscr{P}(\Lambda)$ if there exists some $i \in \mathbb{N}$ such that $X \neq S_{N_{i}}^{V}(X)$. Let the nonempty $M \subset \mathscr{P}(\Lambda)$ be the set of all significant members of $\mathscr{P}(\Lambda)$.

DEFINITION 5.2. Suppose that we have a nonempty finite set $\left\{C_{1}, \ldots, C_{m}\right\}$ of general consequence operators, each defined on a language $L_{i}, 1 \leq i \leq m$ where, at least, one member is axiomless. Define the operator $\Pi C_{m}$ as follows: for any $X \subset L_{1} \times \cdots \times L_{m}$, using the projection $\operatorname{pr}_{i}, 1 \leq i \leq m$, define $\Pi C_{m}(X)=C_{1}\left(\operatorname{pr}_{1}(X)\right) \times \cdots \times C_{m}\left(\operatorname{pr}_{m}(X)\right)$.

(Note that if $\varnothing \neq X \subset L_{1} \times \cdots \times L_{m}$, then for each $i, \operatorname{pr}_{i}(X) \neq \varnothing$. The converse also holds. For the case that $X=\varnothing$, since no $L_{i}$ is empty, choose for each projection the only function that exists with empty domain and nonempty codomain, the empty function. Each of these projections has an empty range [1, page 11].)

THEOREM 5.3. The operator $\Pi C_{m}$ defined on the subsets of $L_{1} \times \cdots \times L_{m}$ is an axiomless consequence operator. If each $C_{i}$ is finitary, then $\Pi C_{m}$ is finitary. Otherwise, $\Pi C_{m}$ is, at least, a general consequence operator.

Proof. (a) Let $X \subset L_{1} \times \cdots \times L_{m}$. Then for each $i, 1 \leq i \leq m, \operatorname{pr}_{i}(X) \subset C_{i}\left(\operatorname{pr}_{i}(X)\right) \subset$ $L_{i}$. But, $X \subset \operatorname{pr}_{1}(X) \times \cdots \times \operatorname{pr}_{m}(X) \subset C_{1}\left(\operatorname{pr}_{1}(X)\right) \times \cdots \times C_{m}\left(\operatorname{pr}_{m}(X)\right) \subset L_{1} \times \cdots \times L_{m}$. Suppose that $X \neq \varnothing$, then $\varnothing \neq \Pi C_{m}(X)=C_{1}\left(\operatorname{pr}_{1}(X)\right) \times \cdots \times C_{m}\left(\operatorname{pr}_{m}(X)\right) \subset L_{1} \times \cdots \times$ $L_{m}$. Hence, $\varnothing \neq \operatorname{pr}_{i}\left(\Pi C_{m}(X)\right)=C_{i}\left(\operatorname{pr}_{i}(X)\right), 1 \leq i \leq m$, implies that $C_{i}\left(\operatorname{pr}_{i}\left(\Pi_{m}(X)\right)\right)=$ $C_{i}\left(C_{i}\left(\operatorname{pr}_{i}(X)\right)\right)=C_{i}\left(\operatorname{pr}_{i}(X)\right), 1 \leq i \leq m$. Hence, $\Pi C_{m}\left(\Pi C_{m}(X)\right)=\Pi C_{m}(X)$. Let $X=$ $\varnothing$. Then each $\operatorname{pr}_{i}(X)=\varnothing$. But there is some $j$ such that $C_{j}$ is axiomless. Hence, $C_{j}\left(\operatorname{pr}_{j}(X)\right)=\varnothing$ implies that $C_{1}\left(\operatorname{pr}_{1}(X)\right) \times \cdots \times C_{m}\left(\operatorname{pr}_{m}(X)\right)=\varnothing$ implies that 
$C_{j}\left(\operatorname{pr}_{j}\left(C_{j}\left(\operatorname{pr}_{j}(X)\right)\right)\right)=\varnothing$. Consequently,

$$
C_{1}\left(\operatorname{pr}_{1}\left(C_{1}\left(\operatorname{pr}_{1}(X)\right)\right)\right) \times \cdots \times C_{m}\left(\operatorname{pr}_{m}\left(C_{m}\left(\operatorname{pr}_{m}(X)\right)\right)\right)=\varnothing
$$

Thus, $\Pi C_{m}\left(\Pi C_{m}(X)\right)=\varnothing$ and (i) holds. Also, $\Pi C_{m}$ is axiomless.

(b) Let $X \subset Y \subset L_{1} \times \cdots L_{m}$. If $X=\varnothing$, then $\varnothing=\Pi C_{m}(X) \subset \Pi C_{m}(Y)$. Suppose that $X \neq \varnothing$, then for each $i, \operatorname{pr}_{i}(X) \subset \operatorname{pr}_{i}(Y)$. Hence, $C_{i}\left(\operatorname{pr}_{i}(X)\right) \subset C_{i}\left(\operatorname{pr}_{i}(Y)\right)$. Therefore, $\Pi C_{m}(X)=C_{1}\left(\operatorname{pr}_{1}(X)\right) \times \cdots \times C_{m}\left(\operatorname{pr}_{m}(X)\right) \subset C_{1}\left(\operatorname{pr}_{1}(Y)\right) \times \cdots \times C_{m}\left(\operatorname{pr}_{m}(Y)\right)=$ $\Pi C_{m}(Y)$ and (ii) holds.

(c) Assume that each $C_{i}$ is finitary and let $x \in \Pi C_{m}(X)$ for nonempty $X$. Then for each $i, \operatorname{pr}_{i}(x) \in C_{i}\left(\operatorname{pr}_{i}(X)\right)$. Since each $C_{i}$ is finitary, then there is some finite $F_{i} \subset$ $\operatorname{pr}_{i}(X)$ such that $\operatorname{pr}_{i}(x) \in C_{i}\left(F_{i}\right) \subset C_{i}\left(\operatorname{pr}_{i}(X)\right)$. Finite $F=F_{1} \times \cdots \times F_{m} \subset \operatorname{pr}_{1}(X) \times$ $\cdots \times \operatorname{pr}_{m}(X)$. Then for each $i, \operatorname{pr}_{i}(F)=F_{i}$ implies that finite $F=F_{1} \times \cdots \times F_{m}=$ $\operatorname{pr}_{1}(F) \times \cdots \times \operatorname{pr}_{m}(F) \subset \operatorname{pr}_{1}(X) \times \cdots \times \operatorname{pr}_{m}(X)$. From (ii), $x \in \Pi C_{m}(F)=C_{1}\left(F_{1}\right) \times \cdots \times$ $C_{m}\left(F_{m}\right) \subset \Pi C_{m}\left(\operatorname{pr}_{1}(X) \times \cdots \times \operatorname{pr}_{m}(X)\right)=C_{1}\left(\operatorname{pr}_{1}(X)\right) \times \cdots \times C_{m}\left(\operatorname{pr}_{m}(X)\right)=\Pi C_{m}(X)$. This completes the proof.

In what follows, consider all of the previously defined notions but only with respect to this informal $V$ and the language $\Lambda$. (Although, the consequence operators are being restricted to a special collection that is of interest to various science-communities, Theorem 5.4 will hold, with obvious modifications, for any sequentially represented set of consequence operators.) Although strictly not necessary, in order for the following results to correlate with the results in [7], embed all of these informal results into the formal superstructure $M=\langle\mathscr{R}, \in,=\rangle$ as done in [4, page 70] where $\mathscr{R}$ is isomorphic to the real numbers. Further, consider the structure $* \mathcal{M}=\left\langle{ }^{*} \mathscr{R}, \in,=\right\rangle$ a nonstandard and elementary extension of $\mathcal{M}$ that is a $2^{|\mathcal{M}|}$-saturated enlargement $(|\cdot|$ denotes cardinality). Finally, consider the superstructure y, the Extended Grundlegend Structure.

A unifying consequence operator approach seems at first to be rather obvious. In actual physical practice, a set of physical theories is applied to a specific $W \subset \Lambda$. The result $C(W)=\bigcup\left\{S_{N_{j}}^{V}(W) \mid j \in \mathbb{N}\right\}$ is most certainly a unification for all of the physical science theories $S_{N_{j}}$. But, define the consequence operator $B$ on $\mathscr{P}(\{A, B, C, D, E\})$ by two relations $(A, B, C),(D, E)$. Then $B(\{A, B\})=\{C\}$ and $B(\{D\})=\{E\}$. Define $R$ on $\mathscr{P}(\{A, B, C, D, E\})$ by one relation $(A, B, D)$. Hence, $R(\{A, B\})=\{D\}$. The union operator $K$ is defined for each $X \in \mathscr{P}(\{A, B, C, D, E\})$ by $K(X)=B(X) \cup R(X)$. However, $K(\{A, B\})=\{A, B, C, D\}$ and $K(K(\{A, B\}))=\{A, B, C, D, E\}$ and (i) does not hold. The union operator, even in the simplest sense, is not a consequence operator. How can this actual physical practice be considered as a rational process? This is done by adjoining the choice aspect of intelligence to the process. Applying this additional step, there is a standard consequence operator styled unification and more than one ultralogic styled unification.

The following ultralogic styled unification is consistent with the modeling of the ultralogic generation of probabilistic behavior [7, 5] and minimal in the hyperfinite sense. In ultralogic theory, attempts are made to stay within the bounds of the "finite" or "hyperfinite." In all known cases, the set of hypotheses selected from $M$ is a 
finite set. For such selections of finite sets of significant hypotheses, the standard consequence operator $P$ used to establish Theorem 5.4 is a finitary consequence operator. It is a practical consequence operator.

THEOREM 5.4. Given the language $\Lambda$ and the sequentially represented set of consequence operators $\left\{S_{N_{j}}^{V} \mid j \in \mathbb{N}\right\}$.

(i) There exists an injection $\mathscr{S}$ on nonempty $M$, the set of all significant subsets of $\Lambda$, into $*(\mathscr{C}(\Lambda))$ such that for each $W \in M, \mathscr{S}_{W}$ is a hyperfinite consequence operator, an ultralogic, such that $\bigcup\left\{\mathbf{S}_{\mathbf{N}_{j}}^{\mathbf{V}}(\mathbf{W}) \mid j \in \mathbb{N}\right\} \subset \bigcup\left\{{ }^{*} \mathbf{S}_{\mathbf{N}_{j}}^{\mathbf{V}}(* \mathbf{W}) \mid j \in \mathbb{N}\right\}=\bigcup\left\{*\left(\mathbf{S}_{\mathbf{N}_{j}}^{\mathbf{V}}(\mathbf{W})\right) \mid j \in\right.$ $\mathbb{N}\} \subset \mathscr{Y}_{W}(* \mathbf{W})$ and $\bigcup\left\{\mathbf{S}_{\mathbf{N}_{j}}^{\mathbf{V}}(\mathbf{W}) \mid j \in \mathbb{N}\right\}=\mathscr{S}_{W}(* \mathbf{W}) \cap \Lambda$.

(ii) If $\varnothing \neq\left\{W_{1}, \ldots, W_{m}\right\} \subset M$, then there exists a hyperfinite consequence operator $\Pi$ defined on internal subsets of the product set ${ }^{*} \Lambda^{m}$ such that for each $i=$ $1, \ldots, m, \mathscr{Y}_{W_{i}}\left({ }^{*} \mathbf{W}_{i}\right)={ }^{*} \operatorname{pr}_{i}\left(\Pi\left({ }^{*} \mathbf{W}_{1} \times \cdots \times{ }^{*} \mathbf{W}_{m}\right)\right)$. If each $W_{i}, 1 \leq i \leq m$, is finitary, then $\Pi$ is hyperfinitary.

(iii) For each $W, A \in M$, if $W \subset A$ then $\mathscr{S}_{W}\left({ }^{*} \mathbf{W}\right) \subset \mathscr{S}_{W}\left({ }^{*} \mathbf{A}\right) \subset \mathscr{I}_{A}\left({ }^{*} \mathbf{A}\right)$.

Proof. (i) In [6, page 4], special sets of consequence operators are defined. For this application and for a given $X \in M$, the set is $H_{X}=\{P(Y, X) \mid Y \subset \Lambda\}$. Each of the consequence operators in $H_{X}$ is defined as follows: for each $Z \subset \Lambda, P(Y, X)(Z)=Y \cup Z$, if $X \subset Z$; and $P(Y, X)(Z)=Z$ otherwise. It is shown in [6] that $P$ is a general consequence operator; if $X$ is nonempty, then $P$ is axiomless; if $X$ is finite, then $P$ is finitary. Let $\varnothing \neq \mathscr{A} \subset \mathscr{P}(\Lambda)$. Suppose that $X \subset Z$, then $P(\cup \mathscr{A}, X)(Z)=(\cup \mathscr{A}) \cup Z=\bigcup\{A \cup Z \mid$ $A \in \mathscr{A}\}=\bigcup\{P(A, X)(Z) \mid A \in \mathscr{A}\}$. Now suppose that $X \not \subset Z$, then $P(\cup \mathscr{A}, X)(Z)=Z=$ $\bigcup\{P(A, X)(Z) \mid A \in \mathscr{A}\} \in H_{X}$. Thus $H_{X}$ is closed under the arbitrary union operator.

Consider the entire set of intrinsic consequence operators $\left\{S_{N_{j}}^{V} \mid j \in \mathbb{N}\right\}$. Define by induction, with respect to the sequentially represented $\left\{S_{N_{i}}^{V} \mid j \in \mathbb{N}\right\}, P_{1}=P\left(S_{N_{1}}^{V}(X), X\right)$, $P_{2}=P\left(S_{N_{1}}^{V}(X) \cup S_{N_{2}}^{V}(X), X\right), \ldots, P_{n}=P\left(S_{N_{1}}^{V}(X) \cup \cdots \cup S_{N_{n}}^{V}(X), X\right)$. From this definition, it follows that for any $n \in \mathbb{N}$ the relation

$$
P_{n}(X)=S_{N_{1}}^{V}(X) \cup \cdots \cup S_{N_{n}}^{V}(X)
$$

holds for each $X \subset \Lambda$. The $P_{n}$, therefore, unify the finite partial sequences of $\left\{S_{N_{j}}^{V} \mid j \in\right.$ $\mathbb{N}$ \}. This restriction to but finite unions is the aspect that allows for a type of minimal ultralogic to be generated.

All of the above is now embedded into $M$ and then considered as embedded into the superstructure $\mathscr{Y}$. Since $\left\{S_{N_{i}}^{V}\right\}$ is sequentially represented, there is a fixed sequence $g$ such that $g(i)=\mathbf{S}_{\mathbf{N}_{i}}^{\mathbf{V}}, g[\mathbb{N}]=\left\{\mathbf{S}_{\mathbf{N}_{j}}^{\mathbf{V}} \mid j \in \mathbb{N}\right\}$ and $g(i)(\mathbf{X})=\mathbf{S}_{\mathbf{N}_{i}}^{\mathbf{V}}(\mathbf{X})$. Hence for arbitrary $X \subset \Lambda$, utilizing $g$, the above inductive definition yields a sequence $f_{\mathbf{X}}: \mathbb{N} \rightarrow \mathbf{H}_{\mathbf{X}}$ such that $f_{\mathbf{X}}(j)=\mathbf{P}_{\mathbf{j}}$ and $f_{\mathbf{X}}(j)(\mathbf{X})=\mathbf{P}_{\mathbf{j}}(\mathbf{X})$ and, as embedded into $\mathcal{M}$, relation (5.2) holds.

Let $X \subset \Lambda$, then the following sentence holds in $\mu$ :

$$
\begin{aligned}
\forall x \forall i\left(( x \in \Lambda ) \wedge ( i \in \mathbb { N } ) \longrightarrow \left(x \in f_{\mathbf{X}}(i)(\mathbf{X})\right.\right. \\
\longleftrightarrow \exists j((j \in \mathbb{N}) \wedge(1 \leq j \leq i) \wedge(x \in g(j)(\mathbf{X}))))) .
\end{aligned}
$$

By $*$-transfer, the sentence

$$
\begin{aligned}
\forall x \forall i\left(\left(x \in{ }^{*} \Lambda\right) \wedge\left(i \in{ }^{*} \mathbb{N}\right)\right. & \longrightarrow\left(x \in *^{*}\left(f_{\mathbf{X}}(i)(\mathbf{X})\right)\right. \\
& \left.\left.\longleftrightarrow \exists j\left(\left(j \in{ }^{*} \mathbb{N}\right) \wedge(1 \leq j \leq i) \wedge\left(x \in{ }^{*}(g(j)(X))\right)\right)\right)\right)
\end{aligned}
$$


holds in * ${ }^{*}$. Due to our method of embedding and identification, sentence (5.4) can be re-expressed as

$$
\begin{aligned}
\forall x \forall i\left(\left(x \in{ }^{*} \Lambda\right) \wedge\left(i \in{ }^{*} \mathbb{N}\right)\right. & \longrightarrow\left(x \in{ }^{*} f_{\mathbf{X}}(i)\left({ }^{*} \mathbf{X}\right)\right. \\
& \left.\left.\longleftrightarrow \exists j\left(\left(j \in{ }^{*} \mathbb{N}\right) \wedge(1 \leq j \leq i) \wedge\left(x \in{ }^{*} g(j)\left({ }^{*} \mathbf{X}\right)\right)\right)\right)\right) .
\end{aligned}
$$

Next consider ${ }^{*} f_{\mathbf{X}}:{ }^{*} \mathbb{N} \rightarrow{ }^{*} \mathbf{H}_{\mathbf{X}}$ and any $\lambda \in{ }^{*} \mathbb{N}-\mathbb{N}$. Then hyperfinite ${ }^{*} f_{\mathbf{X}}(\lambda) \in{ }^{*} \mathbf{H}_{\mathbf{X}}$ is a nonstandard consequence operator, an ultralogic, that is, hyperfinite in the sense that it hyperfinitely generates each of the ${ }^{*} \mathbf{P}_{j}$ and it satisfies statement (5.5). Hence, arbitrary $j \in \mathbb{N}$ and $w \in{ }^{*} g(j)\left({ }^{*} \mathbf{X}\right)={ }^{*} \mathbf{S}_{\mathbf{N}_{j}}^{\mathbf{V}}\left({ }^{*} \mathbf{X}\right)={ }^{*}\left(\mathbf{S}_{\mathbf{N}_{j}}^{\mathbf{V}}(X)\right) \subset{ }^{*} \boldsymbol{\Lambda}$ imply that $w \in$ ${ }^{*} f_{\mathbf{X}}(\lambda)\left({ }^{*} \mathbf{X}\right)$ since $1 \leq j<\lambda$. Observe that ${ }^{\sigma}\left(\mathbf{S}_{N_{j}}^{V}(X)\right) \subset *\left(\mathbf{S}_{N_{j}}^{V}(X)\right)$. However, under our special method for embedding ${ }^{\sigma}\left(\mathbf{S}_{\mathbf{N}_{j}}^{\mathbf{V}}(X)\right)=\mathbf{S}_{\mathbf{N}_{j}}^{\mathbf{V}}(X)$, for an arbitrary $\mathbf{X} \subset \Lambda$.

The final step is to vary the $X \in M$. It is first shown that for two distinct $X, Y \in M$ there is an $m \in \mathbb{N}$ such that $P_{m}^{X}=P\left(S_{N_{1}}^{V}(X) \cup \cdots \cup S_{N_{m}}^{V}(X), X\right) \neq P_{m}^{Y}=P\left(S_{N_{1}}^{V}(Y) \cup\right.$ $\left.\cdots \cup S_{N_{m}}^{V}(Y), Y\right)$. Since $X, Y$ are nonempty, distinct and arbitrary, assume that $X \not \subset Y$. Hence there is some $i \in \mathbb{N}$ and $j \in \mathbb{N}$ such that $X \subset S_{N_{i}}^{V}(X) \neq X$ and $Y \subset S_{N_{j}}^{V}(Y) \neq Y$. Consider some $m \in \mathbb{N}$ such that $i, j \leq m$. Let $Y \subset X$, then $P_{m}^{X}(X)=P\left(S_{N_{1}}^{V}(X) \cup \cdots \cup\right.$ $\left.S_{N_{m}}^{V}(X), X\right)(X)=S_{N_{1}}^{V}(X) \cup \cdots \cup S_{N_{m}}^{V}(X) \neq X \subset P_{m}^{X}(X)$. But $P_{m}^{Y}(X)=X \subset P_{m}^{X}(X) \neq X$. Thus $P_{m}^{Y}(X) \neq P_{m}^{X}(X)$. Now further suppose that $Y \not \subset X$, then there is some $y \in Y$ such that $\{y\} \not \subset X$. Since $X \subset X \cup\{y\}$ and $Y \not \subset X \cup\{y\}$, then $P_{m}^{X}(X \cup\{y\})=\left(S_{N_{1}}^{V}(X) \cup\right.$ $\left.\cdots \cup S_{N_{m}}^{V}(X)\right) \cup\{y\}$. Since $Y \not \subset X \cup\{y\}$, then $P_{m}^{Y}(X \cup\{y\})=X \cup\{y\}$. Again since $S_{N_{1}}^{V}(X) \cup \cdots \cup S_{N_{m}}^{V}(X) \neq X$, then $P_{m}^{Y}(X \cup\{y\}) \neq P_{m}^{X}(X \cup\{y\})$. For consequently, $P_{m}^{Y} \neq$ $P_{m}^{X}$. Further, for any (†) $k \in \mathbb{N}, m \leq k, P_{k}^{Y} \neq P_{k}^{X}$. Consider these results formally stated. Then by $*$-transfer, for each distinct pair $\mathbf{X}, \mathbf{Y} \in \mathbf{M}$ there exists some $m \in{ }^{*} \mathbb{N}$ such that ${ }^{*} f_{\mathbf{X}}(m) \neq * f_{\mathbf{Y}}(m)$. Thus for $\mathbf{X}, \mathbf{Y} \in \mathbf{M}, \mathbf{X} \neq \mathbf{Y}, A(\mathbf{X}, \mathbf{Y})=\left\{m \mid\left(m \in{ }^{*} \mathbb{N}\right) \wedge * f_{\mathbf{X}}(m) \neq\right.$ $\left.* f_{\mathbf{Y}}(m)\right\}$ is nonempty. The Axiom of Choice from the general set theory [3, page 2] used to construct $y$ is now applied. Hence, there exists a set $B$, within our structure, containing one and only member from each $A(\mathbf{X}, \mathbf{Y})$.

The internal binary relation $\left\{(x, y) \mid\left(x \in *^{*}\right) \wedge\left(y \in *^{*} \mathbb{N}\right) \wedge(x \leq y)\right\}$ is, from $*$ transfer of $\mathbb{N}$ properties, a concurrent relation with respect to the range $* \mathbb{N}$. Since $* \mathcal{M}$ is a $2^{|\mathcal{M l}|}$-saturated enlargement and $|B|<2^{|\mathcal{M}|}$, there is some $\lambda \in{ }^{*} \mathbb{N}$ such that for each $i \in B, i \leq \lambda$. Considering this $\lambda$ as fixed, then by $*$-transfer of $(\dagger)$, it follows that for any distinct $X, Y \in M^{*} f_{\mathbf{X}}(\lambda) \neq{ }^{*} f_{\mathbf{Y}}(\lambda)$. Since $M$ is injectively mapped onto $\mathbf{M}$, there exists an injection $\mathscr{S}$ on the set $M$ such that each $W \in M, \mathscr{Y}_{W}={ }^{*} f_{\mathrm{W}}(\lambda) \in{ }^{*}(\mathscr{C}(\Lambda))$. Considering the general properties for such an ${ }^{*} f_{\mathrm{W}}(\lambda)$ as discussed above, it follows that $\bigcup\left\{\mathbf{S}_{\mathbf{N}_{j}}^{\mathbf{V}}(\mathbf{W}) \mid j \in \mathbb{N}\right\} \subset \mathscr{Y}_{W}(* \mathbf{W}) \cap \Lambda$.

Now assume that standard $\mathbf{a} \in \mathscr{S}_{W}\left({ }^{*} \mathbf{W}\right)-\bigcup\left\{\mathbf{S}_{\mathbf{N}_{j}}^{\mathbf{V}}(\mathbf{W}) \mid j \in \mathbb{N}\right\}$. (For our identification and embedding, ${ }^{*} \mathbf{a}=\mathbf{a}$.) Then the sentence

$$
\forall x \forall i((x \in \Lambda) \wedge(i \in \mathbb{N}) \wedge x \in g(i)(\mathbf{W}) \longrightarrow x \neq \mathbf{a})
$$

holds in $\mu$ and, hence,

$$
\forall x \forall i\left(\left(x \in{ }^{*} \Lambda\right) \wedge\left(i \in *^{*} \mathbb{N}\right) \wedge x \in{ }^{*} g(i)\left({ }^{*} \mathbf{W}\right) \longrightarrow x \neq \mathbf{a}\right)
$$

holds in $* \mu$. But since $\mathbf{a} \in{ }^{*} f_{\mathrm{W}}(\lambda)\left({ }^{*} \mathbf{W}\right)$, then statement (5.7) contradicts statement (5.5) and this completes the proof of (i). 
(ii) Note that for each $W \in M,{ }^{*} f_{\mathrm{W}}(\lambda)$ is axiomless by $*$-transfer since every member of ${ }^{*} \mathbf{H}_{W}$ is axiomless. Consider nonempty $\left\{W_{1}, \ldots, W_{m}\right\} \subset M$. Use Definition 5.2 and define $\Pi$ on the internal $X \in{ }^{*}\left(\mathscr{P}(\Lambda)^{m}\right)=* \mathscr{P}\left({ }^{*} \Lambda^{m}\right)$ by $\Pi(X)={ }^{*} f_{\mathrm{W}_{1}}(\lambda)\left({ }^{*} \operatorname{pr}_{1}(X)\right) \times$ $\cdots \times{ }^{*} f_{\mathrm{W}_{m}}(\lambda)\left({ }^{*} \mathrm{pr}_{m}(X)\right)$. It is easily seen by $*$-transfer of Theorem 5.3 that $\Pi$ is a hyperfinite ultralogic. Or, more directly, notice that the properties of the projection maps ${ }^{*} \mathrm{pr}_{i}$ on internal sets are the same as the standard projection maps. Since each member of ${ }^{*} \mathbf{H}_{\mathrm{W}_{i}}$ satisfies the $*$-transfer of axioms (i) and (ii) on internal sets, then for ${ }^{*} f_{\mathrm{W}_{i}}(\lambda)$ as appear in the proof of Theorem 5.3 for parts (a) (b). Since the finite Cartesian product of hyperfinite objects is hyperfinite, then $\Pi$ is hyperfinite. Therefore, $\Pi$ is a hyperfinite ultralogic and for each $i, 1 \leq i \leq m,{ }^{*} \operatorname{pr}_{i}\left(\Pi\left({ }^{*} \mathbf{W}_{1} \times \cdots \times{ }^{*} \mathbf{W}_{m}\right)\right)=$ ${ }^{*} f_{\mathrm{W}_{i}}(\lambda)\left({ }^{*} \mathbf{W}_{i}\right)=\mathscr{S}_{W_{i}}\left({ }^{*} \mathbf{W}_{i}\right)$. Finally, if each $W_{i}$ is finitary, then each ${ }^{*} f_{\mathrm{W}_{i}}(\lambda)$ satisfies the $*$-transfer of axiom (3). This means that in the place of the "finite" sets, hyperfinite sets are utilized. Again, since the finite Cartesian product of hyperfinite sets is hyperfinite, then $\Pi$ is hyperfinitary.

(iii) For each $j \in \mathbb{N}, W, A \in M$, where $W \subset A$, it follows that $P_{j}^{W}(W) \subset P_{j}^{W}(A)=$ $S_{N_{i}}^{V}(W) \cup \cdots \cup S_{N_{j}}^{V}(W) \cup A$. But, $P_{j}^{A}(A)=S_{N_{1}}^{V}(A) \cup \cdots \cup S_{N_{j}}^{V}(A) \cup A=S_{N_{1}}^{V}(A) \cup \cdots \cup$ $S_{N_{j}}^{V}(A)$. Since for each $i \in \mathbb{N}, S_{N_{i}}^{V}(W) \subset S_{N_{i}}^{V}(A)$, then $P_{j}^{W}(A) \subset P_{j}^{A}(A)$. Thus, after embedding, $f_{\mathrm{W}}(j)(\mathbf{W}) \subset f_{\mathrm{W}}(j)(\mathbf{A}) \subset f_{\mathrm{A}}(j)(\mathbf{A})$. By $*$-transfer, this yields that $\mathscr{S}_{W}\left({ }^{*} \mathbf{W}\right)=$ ${ }^{*} f_{\mathrm{W}}(\lambda)\left({ }^{*} \mathbf{W}\right) \subset \mathscr{I}_{W}(* \mathbf{A})={ }^{*} f_{\mathrm{W}}(j)\left({ }^{*} \mathbf{A}\right) \subset \mathscr{S}_{A}(* \mathbf{A})={ }^{*} f_{\mathrm{A}}(j)\left({ }^{*} \mathbf{A}\right)$. This completes the proof.

COROLLARY 5.5. If $\left\{S_{N_{j}}^{V} \mid j \in \mathbb{N}\right\}$ represents all of the physical theories that describe natural world behavior, then the choice function and the last equation in part (i) of Theorem 5.4 and part (ii) correspond to an ultralogic unification for $\left\{S_{N_{j}}^{V} \mid j \in \mathbb{N}\right\}$.

REMARK 5.6. Obviously, the results of Theorem 5.4(i), (iii) and Corollary 5.5(i) do not require that the standard theory consequence operators be axiomless. Further, in proofs such as that of Theorem 5.4, some of the results can be obtained without restricting the construction to members of $H_{X}$. Simply define, for $X \subset \Lambda, P_{n}^{\prime}(X)=$ $S_{N_{1}}^{V}(X) \cup \cdots \cup S_{N_{n}}^{V}(X)$. One obtains a hyperfinite ${ }^{*} h_{\mathrm{W}}(\lambda)$ from this definition. The use of the $H_{X}$ objects is to further identify ${ }^{*} f_{\mathrm{W}}(\lambda)$ as an ultralogic that is also hyperfinite and of the type used in $[7,5]$ and, hence, allows for the modeling of certain aspects of intelligence. In general, a basic aspect of intelligence is to select a specific member of $M$ to which to apply a specific physical theory while rejecting other members of $M$ as applicable. This special aspect of intelligence is not usually modeled. But, this additional aspect of intelligence is modeled by the choice procedures in the proof of Theorem 5.4 and the specific selection of a member of $H_{X}$.

Of significance is that Corollary 5.5 is technically falsifiable. The most likely falsifying entity would be the acceptance of a physical theory that does not use the rules of inference as setout in Section 3. In particular, when different hypotheses are considered, the requirement that the rules of inference RI cannot be altered.

6. A standard consequence operator unification. In practical science, each $S_{N_{j}}^{V}$ is applied to a finite $X \subset \Lambda$. In [6, Theorem 5.1], is the same as Theorem 5.4 part (i) in this paper. There is a major difference, however, in how the results are obtained. In 
Theorem 5.4, a considerably different standard consequence operator $P$ is used. Since, in general, the union operator does not generate a consequence operator, any unifying consequence operator standard or nonstandard would have additional characteristics. These characteristics considered from the physical theory viewpoint might be rather undesirable. For example, the standard consequence operator $C_{m}^{X}$ used in [6, Theorem 5.1] when applied at any nonempty $Y \subset X$, where $Y, X \in M$, has the property that $C_{m}^{X}(Y)=C_{m}^{X}(X)=S_{N_{1}}^{V}(X) \cup, \ldots, \cup S_{N_{m}}^{V}(X)$. Although the choice function does not allow an application to objects distinct from $X$, the fact that $C_{m}^{X}$ satisfies the consequence operator axioms does require such an application. Can such necessary secondary requirements be ignored based upon practical physical usage? What actually is assumed to occur is that such a unification is applied throughout the entire universe at each moment in cosmic time. Under the assumption that all scientific theories must be consistent in combined form, then a practical union operator should share the same rationality as the individual $S_{N}^{V}$ operators.

THEOREM 6.1. Define for each $X \in \mathscr{P}(\Lambda)$, the operator $P_{\infty}^{X}=P\left(\bigcup\left\{S_{N_{j}}^{V}(X) \mid j \in\right.\right.$ $\mathbb{N}\}, X) \in H_{X}$.

(i) There is an injection $S$ on $M$ such that for each $W \in M, S_{W}=P_{\infty}^{W}$.

(ii) For each $W \in M$, and $S_{W}(W)$, the set $S_{W}(A), A \neq W, A \in M$, is consistent with the alterations in natural-system behavior modeled by $S_{W}(W)$. Further, if $W \subset A$, then $S_{W}(W) \subset S_{W}(A) \subset S_{A}(A)$.

(iii) If $\varnothing \neq\left\{W_{1}, \ldots, W_{m}\right\} \subset M$, then there exists a consequence operator $\Pi$ defined on $\mathscr{P}\left(\Lambda^{m}\right)$ such that for each $i=1, \ldots, m, S_{W_{i}}\left(W_{i}\right)=\operatorname{pr}_{i}\left(\Pi\left(W_{1} \times \cdots \times W_{m}\right)\right)$. If each $W_{i}$, $1 \leq i \leq m$, is finitary, then $\Pi$ is finitary.

(iv) For each $W \in M, \mathscr{S}_{W}\left({ }^{*} \mathbf{W}\right) \subset{ }^{*} \mathbf{S}_{\mathrm{W}}\left({ }^{*} \mathbf{W}\right)$.

Proof. (i) The proof in Theorem 5.4 that shows that for distinct $W, Y \in M, P_{m}^{W} \neq$ $P_{m}^{Y}$ also holds for $P_{\infty}^{W}$ and $P_{\infty}^{Y}$. Hence, simply define a map $S$ on $M$ by $S_{W}=P_{\infty}^{W}$. This map is an injection.

(ii) If $W \in M$, then $P_{\infty}^{W}(W)=\bigcup\left\{S_{N_{j}}^{V}(W) \mid j \in \mathbb{N}\right\}$ follows from the definition of the $P$ consequence operator. Now assume that $A \in M, W \neq A$. First, let $W \subset A$, thus $W$ represents a subsystem of the natural-system $A$ that is distinct from $A$. Then $P_{\infty}^{W}(W)(A)=\bigcup\left\{S_{N_{j}}^{V}(W) \mid j \in \mathbb{N}\right\} \cup A$. Is this adjoined $A$ of any physical concern? Since $W \subset A$ is a very general statement without any other characterizing requirements, it is not known whether $A \subset \bigcup\left\{S_{N_{j}}^{V}(W) \mid j \in \mathbb{N}\right\}$. Thus the results can be interpreted as stating that when $P_{\infty}^{W}$ is applied to a subsystem $W \subset A$ the result contains those members $A$ that are not altered by any of the $S_{N_{j}}^{V}$ applied to $W$. Of course, one may also assume that at the same instant $P_{\infty}^{A}$ is applied to $A$. This yields that $P_{\infty}^{W}(W) \subset P_{\infty}^{A}(A)$ which is exactly as one would expect. In the case that $W \not \subset A$, then $P_{\infty}^{W}(A)=A$. This is consistent with the lack of a specific characterizing sharing relation between the $W$ and $A$ natural-systems. Of course, $P_{\infty}^{A}\left(P_{\infty}^{W}(A)\right)=P_{\infty}^{A}(A)=\bigcup\left\{S_{N_{j}}^{V}(A) \mid j \in \mathbb{N}\right\}$. Thus neither of these results appears to have any significant physical inconsistencies. The statement that if $W \subset A$, then $S_{W}(W) \subset S_{W}(A) \subset S_{A}(A)$ is established in the same manner as part (iii) of Theorem 5.4.

(iii) Theorem 5.3. 
(iv) From Theorem 5.4, for each $W \in M, \mathscr{S}_{W}\left({ }^{*} \mathbf{W}\right)={ }^{*} f_{\mathrm{W}}(\lambda)\left({ }^{*} \mathbf{W}\right)$. From the definition of the sequence $f$ the following

$$
\forall i \forall x\left((x \in \Lambda) \wedge(i \in \mathbb{N}) \wedge\left(x \in f_{\mathrm{W}}(i)(\mathbf{W})\right) \rightarrow x \in \mathrm{S}_{\mathrm{W}}(\mathbf{W})\right)
$$

holds in $M$. Hence, by $*$-transfer

$$
\forall i \forall x\left(\left(x \in{ }^{*}\right) \Lambda \wedge\left(i \in{ }^{*} \mathbb{N}\right) \wedge\left(x \in{ }^{*} f_{\mathrm{W}}(i)\left({ }^{*} \mathbf{W}\right)\right) \longrightarrow x \in{ }^{*} \mathbf{S}_{\mathrm{W}}\left({ }^{*} \mathbf{W}\right)\right)
$$

However, letting $i=\lambda$ and noting that ${ }^{*} f_{\mathrm{W}}(\lambda)\left({ }^{*} \mathbf{W}\right)=\mathscr{S}_{W}\left({ }^{*} \mathbf{W}\right)$, then this completes the proof.

REMARK 6.2. From $*$-transfer, the basic results in Theorem 6.1(ii) also hold for ${ }^{*} \mathbf{S}_{\mathrm{W}}\left({ }^{*} \mathbf{W}\right)$ and ${ }^{*} \mathbf{S}_{\mathrm{A}}\left({ }^{*} \mathbf{A}\right)$ as well as internal arguments in general.

\section{REFERENCES}

[1] J. Dugundji, Topology, Series in Advanced Mathematics, Allyn and Bacon, Massachusetts, 1966. MR 33\#1824. Zbl 144.21501.

[2] R. A. Herrmann, Nonstandard consequence operators, Kobe J. Math. 4 (1987), no. 1, 1-14, http://www.arXiv.org/abs/math.LO/9911204. MR 89d:03068. Zbl 639.03070.

[3] _ , The theory of ultralogics part I, http://www.arXiv.org/abs/math.GM/9903081, 1999.

[4] _ The theory of ultralogics part II, http://www.arXiv.org/abs/math.GM/9903082, 1999.

[5] _ The wondrous design and non-random character of "chance" events, http://www. arXiv.org/abs/physics/9903038, 1999.

[6] _ An ultralogic unification for all physical theories, http://www.arXiv.org/abs/ physics/0101009, 2001.

[7] R. A. Herrmann, Ultralogics and probability models, Int. J. Math. Math. Sci. 27 (2001), 321325.

[8] A. Tarski, Logic, Semantics, Metamathematics. Papers from 1923 to 1938, Clarendon Press, Oxford, 1956. MR 17,1171a. Zbl 075.00702.

ROBERT A. HerRmanN: MATHEMATICS DEPARTMENT, U. S. NAVAL ACADEMY, 572C Holloway RD, ANNAPOLIS, MD 21402-5002, USA

E-mail address: rah@usna.edu 


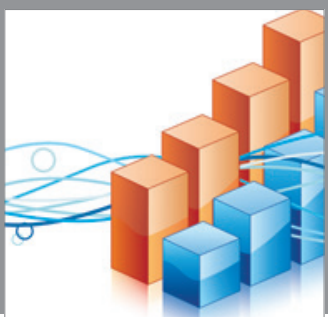

Advances in

Operations Research

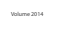

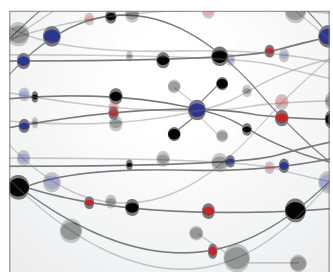

\section{The Scientific} World Journal
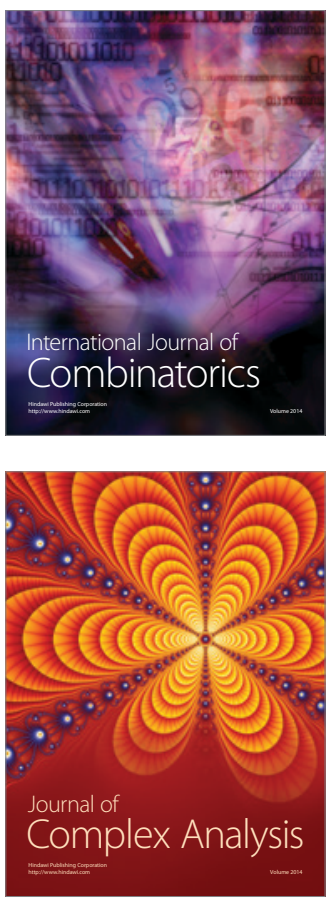

International Journal of

Mathematics and

Mathematical

Sciences
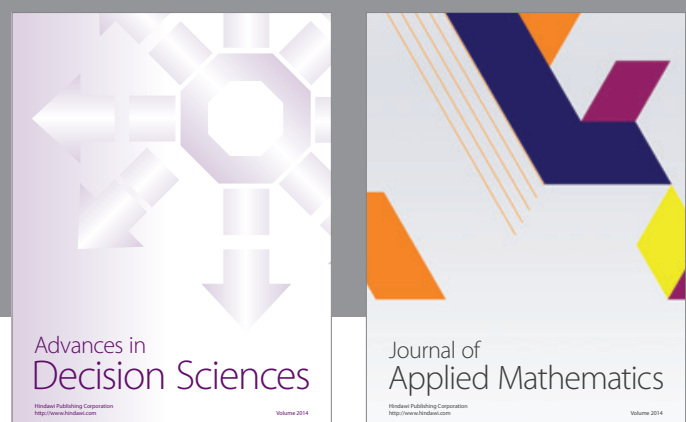

Journal of

Applied Mathematics
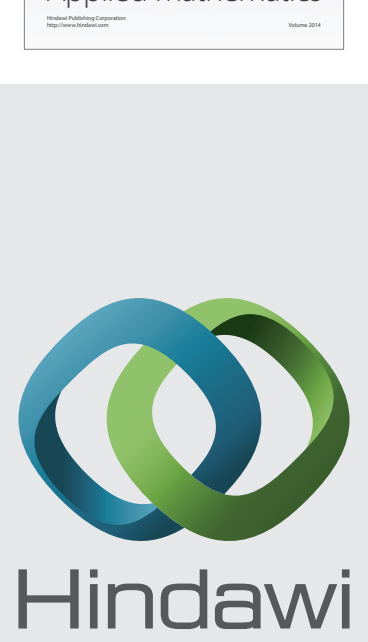

Submit your manuscripts at http://www.hindawi.com
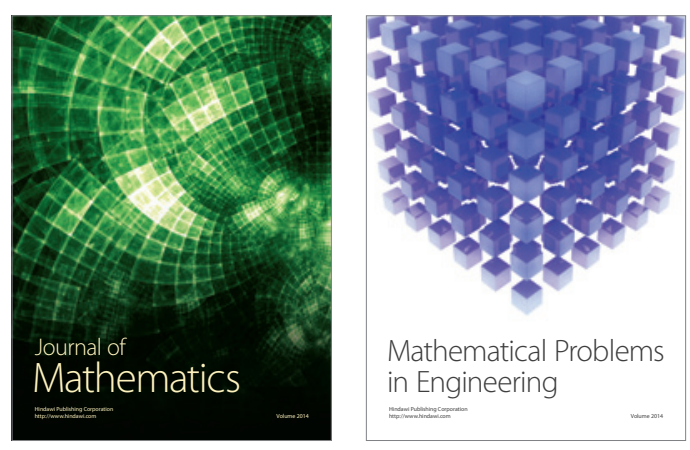

Mathematical Problems in Engineering
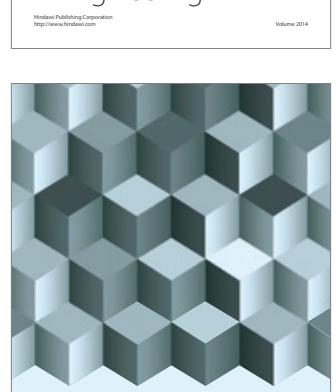

Journal of

Function Spaces
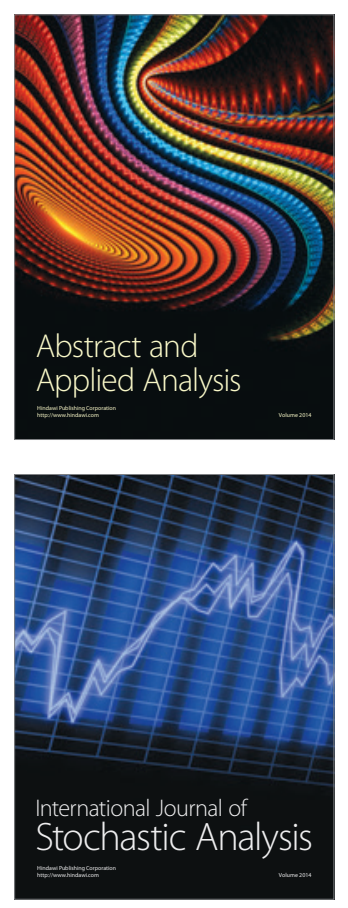

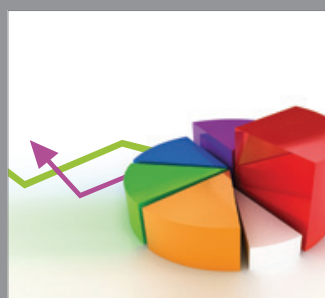

ournal of

Probability and Statistics

Promensencen
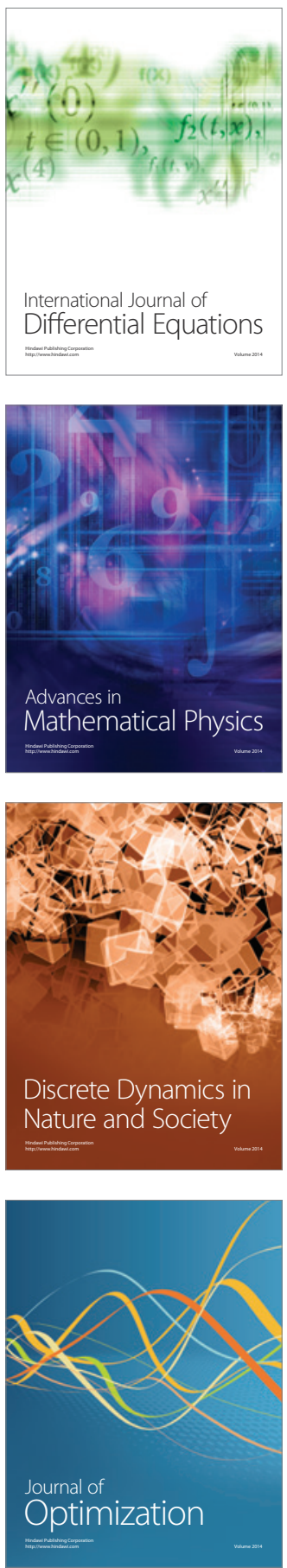